\title{
Shigella flexneri-induced vaginitis in a prepubertal children: description of a case
}

\author{
Antonella Restelli, Manuela Colosimo, Anna Maraschini, Anna Grancini, Rosaria Colombo, \\ Romualdo Grande, Maria Rosa Araldi, Erminio Torresani \\ Laboratorio Centrale di Analisi Chimico-Cliniche e Microbiologia, Fondazione IRCCS Ca'-Granda Ospedale Maggiore \\ Policlinico
}

Key words: Vaginitis, Shigella, S. flexneri, Prepubertal age

Descrizione di un caso di vulvovaginite da S. flexneri in età prepubere

\section{SUMMARY}

In prepuberal girls vulvo-vaginitis are caused by germs of intestinal origin, mycetes, Gardnerella vaginalis, protozoa. Shigella is an uncommon agent able to induce valvovaginitis in children.

We report the case of a 7-year-old girl with chronic vulvo-vaginitis caused by S. flexneri.

Antibiotic Susceptibility Testing revealed that S. flexnery was sensible to cefotaxime, amoxicillin, imipenem, ciprofloxacin, but resistant to amikacin, cefazolin, gentamycin, ampicillin and tetracycline.

A treatment with ciprofloxacin brought to a rapid resolution of all symptoms.

At the follows up at 3 and 6 months the patient did not report symptoms of infection or articular cartilage abnormality; microbiological evaluations were also negative.

Even if it is a single case report and other clinical trial may be performed in order to validate this hypothesis, we speculate that in patient with vulvo-vaginal infection living in environment with low hygiene care, a carefully microbiological evaluation of uncommon agents may be performed.

\section{INTRODUZIONE}

Il rivestimento vulvo-vaginale delle bambine in età prepubere presenta, rispetto all'adulto, specifiche caratteristiche differenziative: mucosa più sottile, priva di flora saprofita predominante, e $\mathrm{pH}$ neutro (7-8). La colonizzazione da parte di batteri intestinali o la contaminazione ad opera di microrganismi presenti nell'ambiente esterno, è relativamente frequente; comunque, sono comuni anche le reazioni irritative o allergiche secondarie all'uso di detergenti, disinfettanti, profumi, fibre sintetiche e alla presenza di residui di detersivi usati per il lavaggio degli indumenti.

Le vulvovaginiti microbiche possono avere diversa patogenesi:

- aspecifiche, statisticamente più frequenti, e sostenute da flora polimicrobica, o correlate ad infestazioni da ossiuri o legate alla presenza di corpi estranei.

- specifiche, meno frequenti e causate da microrganismi patogeni (Escherichia coli, Streptococcus pyogenes, Haemophilus influenzae, Haemophilus parainfluenzae, Staphylococcus aureus e, più raramente, lieviti del genere Candida) che giungono alla regione vulvovaginale dall'apparato gastrointestinale, dalla cute, dall'apparato urinario o per via ematica (6) (Tabella 1).

Clinicamente le vulvovaginiti si manifestano con iperemia vulvare, secrezione purulenta, e talora anche francamente ematica (6-11). In pazienti con situazioni socio-economiche disagiate sono stati descritti casi di infezioni sostenute da Shigella (9$10)$ e, tra le varie specie, $S$. flexneri rappresenta l'agente infettante più frequente: non sempre tale microrganismo è riscontrabile nelle coprocolture e a volte queste forme tendono a cronicizzarsi (3). Descrizione del caso

Scopo del nostro lavoro è stato quello di descrivere il caso di una bambina con una vulvovaginite sostenuta da $S$. flexneri.

Una bambina di 7 anni, nata e vissuta in Ecuador, è giunta in Italia nel settembre 2006, alcuni mesi più tardi ha accusato dolori inguinali e vulvari con presenza di secrezioni vaginali prima giallastre e poi ematiche.

La visita ginecologica effettuata presso il nostro ambulatorio ha evidenziato la presenza di perdite ematiche vaginali, dolorabilità addominale e sospetto abuso sessuale, per cui la bambina è stata inviata presso il soccorso violenze sessuali. Il colloquio con la madre ha portato all'esclusione dell'abuso sessuale.

Nel giugno 2008 a seguito della persistenza della sintomatologia dolorosa e delle perdite siero-ematiche vaginali, la bambina giunge nuovamente all'osservazione presso il nostro nosocomio.

\section{Corresponding author: Antonella Restelli}

Via Vergiate 3; 2015I Milano

Tel.: +393476920588 - Fax: +390255033645

E-mail: arestelli@policlinico.mi.it 
Vengono effettuati un tampone vaginale per la ricerca di batteri, miceti e Trichomonas vaginalis che sono risultati negativi. Gli esami ormonali (estradiolo, FSH, LH, TSH, fT3 fT4), erano nella norma mentre gli esami ematochimici hanno evidenziato un modico aumento delle transaminasi (AST 78 U/1, ALT 96 U/1), gammaGT 134 U/1, e FA $347 \mathrm{U} / 1$ ed eosinofilia $\left(2.80 \times 10^{3} / \mathrm{mmc}\right)$. L'esame radiografico dell'addome senza mezzo di contrasto e l'ecografia addominale non hanno evidenziato patologie in atto. La vaginoscopia non ha evidenziato presenza di corpi estranei, ma ha documentato una mucosa iperemica di aspetto distrofico tipico di un quadro infiammatorio-infettivo.

La ricerca di parassiti fecali ha evidenziato la presenza di Endolimax nana e di Dientamoeba fragilis per cui la paziente ha iniziato una terapia con metronidazolo.

La persistenza delle perdite siero-ematiche e della sintomatologia dolorosa hanno portato all'esecuzione di ulteriori indagini microbiologiche estendendo le indagini colturali alla ricerca di batteri meno comuni.

La coltura del secreto vaginale, effettuata seminando il campione su piastre di Agar cioccolato, Agar Columbia CNA, Agar MacConkey, Sabouraud + Cloramfenicolo e incubando a $35^{\circ} \mathrm{C}$ per 24 - $48 \mathrm{~h}$, ha evidenziato la crescita di colonie non fermentanti il lattosio su piastre di Agar MacConkey.

L'identificazione biochimica e l'antibiogramma con sistema VITEK 2 (bioMérieux) hanno evidenziato la presenza di $S$. flexneri sensibile ad amoxicillina-acido clavulanico, cotrimoxazolo, cefalosporine di III generazione, imipenem, piperacillina-tazobactam, ciprofloxacina, e resistente ad amikacina, cefazolina, gentamicina, ampicillina e tetraciclina.

La bambina ha iniziato un trattamento antibiotico con cefotaxime (100 mg/kg/die per 15 giorni), ma la comparsa di un rush cutaneo dopo 7 giorni ne ha obbligato la sospensione.

Il persistere della sintomatologia e delle secrezioni ha indotto la sostituzione della cefalosporina con piperacillina-tazobactam $(100 \mathrm{mg}+12.5 \mathrm{mg}$ ogni 8 ore per 7 giorni), ma, anche in questo caso dopo alcuni giorni viene sospesa per la comparsa di una reazione allergica. La positività del tampone di controllo ha portato alla decisione di iniziare una ulteriore terapia con ciprofloxacina $(25 \mathrm{mg} / \mathrm{kg} / \mathrm{die}$ per 15 giorni) in associazione con estriolo crema per applicazione locale per 12 giorni.

L'esame colturale di controllo effettuato al termine della terapia è risultato negativo e l'esame obiettivo ha evidenziato una normalizzazione della mucosa con remissione delle perdite ematiche e scomparsa della leucorrea.

\section{COMMENTO}

Le vulvovaginiti sono una patologia ginecologica pediatrica abbastanza comune, sostenuta da flora mista o microrganismi intestinali, batteri, funghi, o parassiti. La Shigella è un agente patogeno raro e spesso non riconosciuto come causa di vulvovaginiti in bambine in età prepubere (9-10). Sebbene alcune specie di Shigella siano potenzialmente in grado di indurre tale quadro clinico la maggior parte dei casi descritti in letteratura sono sostenuti da S. flexneri (3).

La facilità di colonizzazione da parte di questo microrganismo è legata alle scarse condizioni di igiene in regione perineale e al $\mathrm{pH}$ vaginale neutro o alcalino, della mucosa che ne favorisce la sopravvivenza (7-8), infatti la bimba viveva in condizioni socio-economiche modeste e scarsamente igieniche.

L'antibiogramma eseguito mediante metodica VITEK 2 ha dimostrato una elevata sensibilità della Shigella nei confronti delle cefalosporine di III generazione e per tale motivo è stata iniziata una terapia con cefotaxime. Le cefalosporine sono antibiotici di solito ben tollerati ed indicati in età pediatrica tuttavia possono indurre reazioni allergiche (5).

Infatti, proprio lo sviluppo di rush cutaneo ha indotto la sospensione del trattamento.

Malgrado recenti lavori abbiano documentato una crescente resistenza delle specie di Shigella nei

Tabella I. Patogenicità dei microrganismi associati a perdite vaginali in età prepubere

\begin{tabular}{ccc}
\hline Patogeni & Probabili patogeni & Non patogeni \\
\hline C. trachomatis* & G. vaginalis* & Lactobacillus \\
\hline T. vaginalis* & M. hominis* & Streptococchi alfa emolitici \\
\hline N. gonorrhoeae* & & \\
\hline Streptococchi beta emolitici & Corinebacterium spp. \\
\hline S. aureus & \\
\hline H. influenzae & \\
\hline Sharainfluenze & \\
\hline Chigella spp. & \\
\hline Candida & \\
\hline Nematodi (ossiuri) & \\
\hline
\end{tabular}


confronti della ciprofloxacina (2-4), nella nostra paziente, l'antibiogramma ha mostrato una elevata sensibilità verso ciprofloxacina.

Tale antibiotico ha, inoltre, una eccellente penetrazione nei tessuti dell'apparato genitale ma può provocare danno articolare nei bambini (1-8).

Nel nostro caso la terapia con ciprofloxacina ha portato ad una completa eradicazione clinica e microbiologica dell'infezione e non ha indotto sviluppo di reazioni avverse locali o sistemiche. In conclusione l'infezione da Shigella dovrebbe essere sospettata anche nelle bambine con vulvovaginite, specialmente se l'anamnesi metta in evidenza scarse condizioni igieniche. In assenza di risposta al trattamento con beta-lattamici o in presenza di reazioni avverse, il trattamento con fluorochinoloni può essere attuato considerando attentamente il rapporto rischi-benefici.

\section{BIBLIOGRAFIA}

1. Baiulescu M, Hannon PR, Marcinak JF, et al. Chronic vulvovaginitis caused by antibiotic-resistant Shigella flexneri in prepuberal child. Pediatr Infect Dis J. 2002; 21 (2): 170-2.

2. Centers for Disease Control and Prevention (CDC). Notes from the field: emergence of Shigella flexneri 2a resistant to ceftriaxone and ciprofloxacin. South Carolina, October 2010. MMWR Morb Mortal Wkly Rep. 2010 Dec 17; 59 (49): 1619

3. Davis TC. Chronic vulvovaginitis in children due to Shigella flexneri. Pediatrics 1975; 56: 41-4.

4. Folster JP, Pecic G, Bowen A, Rickert R, Carattoli A, Whichard JM. Decreased susceptibility to ciprofloxacin among Shigella isolated in the United States 2006-2009. Antimicrob Agents Chemother. 2011 Jan 10.

5. Gallelli L, Ferreri G, Colosimo M, et al. Adverse drug reactions to antibiotics observed in two pulmonology divisions of Catanzaro, Italy: a six-year retrospective study. Pharmacol Res 2002; 46: 395-400.

6. Jacquiery A, Stylianopoulos A, Hogg G, Grover S. Vulvovaginitis: clinical features, aetiology and microbiology of the genital tract. Arch Dis Child 1999; 81: 64-7.

7. Jasper JM, Ward MA. Shigella vulvovaginitis in a prepuberal child. Pediatric Emergency Care 2006; 22: 585-6.

8. Leibovitz E, Janco J, Piglansky L, et al. Oral ciprofloxacin vs. intramuscular ceftriaxone as empiric treatment of acute invasive diarrhoea in children. Pediatr Infect Dis J 2000; 19: 1060-7.

9. Murphy TV, Nelson JD. Shigella vaginitis: Report of 38 patients and review of the literature. Pediatrics 1979; 63: 511-6.

10. Sanders DY, Wasilauskas BL. Shigella vaginitis: Clinical notes on two childhood cases. Clin Pediatr 1973; 12: 54-5. 